\title{
Endothelial Tie1-mediated angiogenesis and vascular abnormalization promote tumor progression and metastasis
}

\author{
Silvia La Porta, ${ }^{1,2}$ Lise Roth, ${ }^{1,2}$ Mahak Singhal,, ${ }^{1,2}$ Carolin Mogler, ${ }^{1,3}$ Carleen Spegg, ${ }^{1}$ Benjamin Schieb, ${ }^{1,2}$ Xianghu Qu, ${ }^{4,5}$ \\ Ralf H. Adams, ${ }^{6,7}$ H. Scott Baldwin,, ${ }^{4,5}$ Soniya Savant, ${ }^{1}$ and Hellmut G. Augustin ${ }^{1,2,8}$ \\ 'Division of Vascular Oncology and Metastasis, German Cancer Research Center (DKFZ-ZMBH Alliance), Heidelberg, Germany. ${ }^{2}$ Department of Vascular Biology and Tumor Angiogenesis (CBTM), Medical \\ Faculty Mannheim, Heidelberg University, Mannheim, Germany. ${ }^{3}$ Institute of Pathology, Technical University Munich, Munich, Germany. ${ }^{4}$ Department of Pediatrics, Division of Cardiology, and ${ }^{5}$ Department \\ of Cell and Developmental Biology, Vanderbilt University School of Medicine, Nashville, Tennessee, USA. ${ }^{6}$ Department of Tissue Morphogenesis, Max Planck Institute for Molecular Biomedicine, Münster, \\ Germany. ${ }^{7}$ Faculty of Medicine, University of Münster, Münster, Germany. ${ }^{8}$ Cerman Cancer Consortium (DKTK), Heidelberg, Germany.
}

The endothelial tyrosine kinase receptor Tie1 remains poorly characterized, largely owing to its orphan receptor status. Clobal Tie1 inactivation causes late embryonic lethality, thereby reflecting its importance during development. Tie1 also plays pivotal roles during pathologies such as atherosclerosis and tumorigenesis. In order to study the contribution of Tie1 to tumor progression and metastasis, we conditionally deleted Tie1 in endothelial cells at different stages of tumor growth and metastatic dissemination. Tie1 deletion during primary tumor growth in mice led to a decrease in microvessel density and an increase in mural cell coverage with improved vessel perfusion. Reduced angiogenesis and enhanced vascular normalization resulted in a progressive increase of intratumoral necrosis that caused a growth delay only at later stages of tumor progression. Concomitantly, surgical removal of the primary tumor decreased the number of circulating tumor cells, reduced metastasis, and prolonged overall survival. Additionally, Tie1 deletion in experimental murine metastasis models prevented extravasation of tumor cells into the lungs and reduced metastatic foci. Taken together, the data support Tie1 as a therapeutic target by defining its regulatory functions during angiogenesis and vascular abnormalization and identifying its role during metastasis.

\section{Introduction}

The high demand for oxygen and nutrients of growing tumors with substantial intratumoral hypoxia gradients stimulates the recruitment of blood vessels through the continuous production of proangiogenic molecules in the tumor milieu (1). Tumor blood vessels grow highly irregular and tortuous, resulting in an abnormal and often poorly functioning vasculature (2-4). The loose association between the endothelium and perivascular cells in the tumor vasculature favors tumor cell intravasation through the vascular wall into the circulation, thereby promoting metastatic spreading to secondary sites (1). The dependence of tumor growth on an adequate supply of blood vessels has stimulated the development of antiangiogenic drugs, most notably compounds targeting the VEGF/VEGFR pathway (5). Antiangiogenic drugs have become part of first-line cancer therapy, however, their efficacy is limited and their mechanism of action is incompletely understood. Originally developed with the goal of starving tumors to death, it is widely recognized today that the clinical efficacy of antiangiogenic drugs results from a combination of partial vascular pruning and normalization of intratumoral

Authorship note: S. La Porta and L. Roth contributed equally as co-first authors. Conflict of interest: The authors have declared that no conflict of interest exists. Submitted: April 19, 2017; Accepted: November 30, 2017

Reference information: / Clin Invest. 2018;128(2):834-845.

https://doi.org/10.1172/JCI94674 microvessels. As a result, the remaining tumor vasculature is better perfused, thereby enabling an improved efficacy of chemotherapeutic agents (6).

The limited efficacy of clinically approved anti-VEGF/VEGFR drugs has stimulated the search for other angiogenesis-regulating pathways whose cotargeting with the VEGF pathway would yield synergistic therapeutic effects. Among these, the angiopoietin/Tie ligand receptor pathway has received particular attention. Ang/ Tie signaling controls later steps of the angiogenic cascade related to vascular maturation and acquisition of the quiescent endothelial cell (EC) phenotype (7). Angiopoietin-1 (Ang1) is paracrine acting and is the primary agonistic ligand of Tie2 (8). Its multimeric structure can bind the Tie2 extracellular domain homodimer for strong Tie2 activation (9).

In turn, Ang2 is produced by ECs and acts in an autocrine manner as a partial Tie2 agonist, i.e., it quenches Tie2 signaling in the presence of Ang1 and weakly stimulates Tie 2 in the absence of Ang1 (10,11). Indeed, because of its dimeric conformation, Ang2 fails to bridge Tie2 extracellular domain homodimers, limiting receptor dimerization and activation (9). As an antagonist of constitutive Ang1/Tie2 signaling, Ang2 acts contextually, enabling angiogenesis in the presence of angiogenic growth factors or driving vessels into regression in the absence of angiogenic stimuli (12). Ang2 is strongly upregulated during tumor angiogenesis and is consequently intensely pursued as an antiangiogenic target (13-15). Yet, recent clinical studies have 


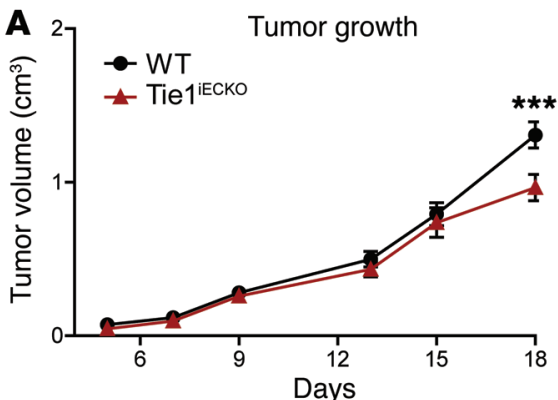

D

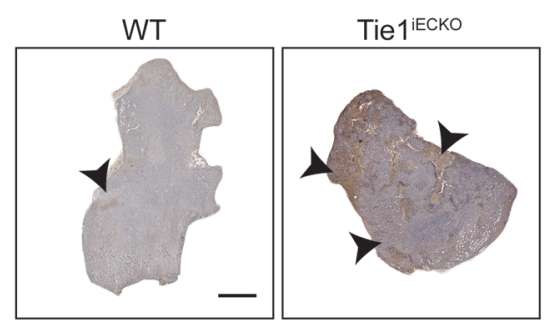

$\mathbf{F}$

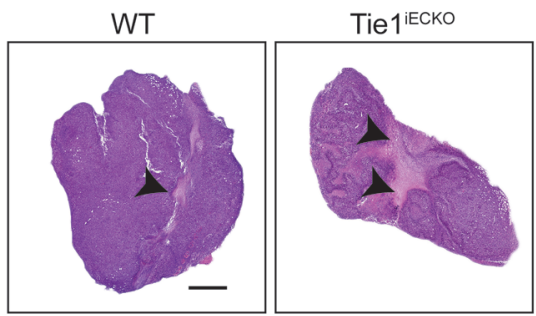

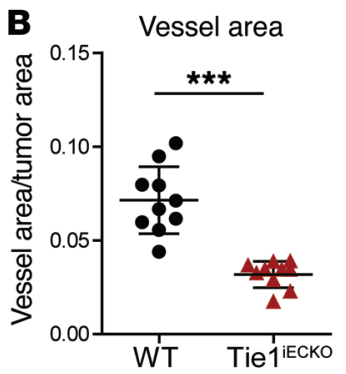
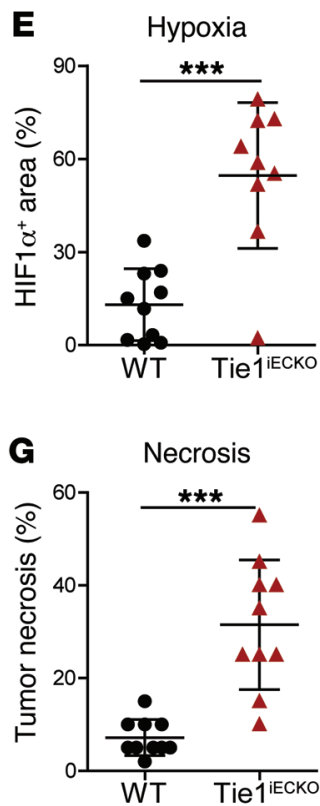

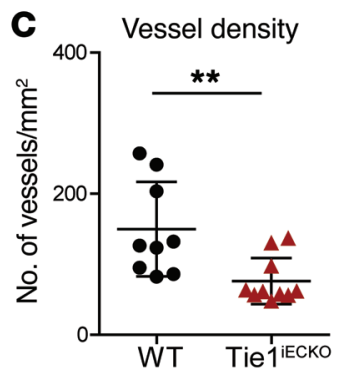

Figure 1. Tie1 loss reduces angiogenesis and delays tumor growth at late stages. (A) Growth curves of LLC tumors in WT and Tie1 $1^{\mathrm{iECKO}}$ mice ( $\left.n=9 \mathrm{WT} ; n=8 \mathrm{Tie}^{\mathrm{iECKO}}\right) .{ }^{* *} P<0.001$, by 2-way ANOVA. Data are expressed as the mean \pm SEM. (B and C) Quantification of vessel area (B) and vessel density (C) in LLC tumors from WT and Tie1 ${ }^{\mathrm{IECKO}}$ mice $(n=9-10)$. ${ }^{* *} P<0.01$ and ${ }^{* *} P<0.001$, by 2 -tailed Mann-Whitney $U$ test. Error bars represent mean \pm SD. (D) Representative images of LLC tumor hypoxia (stained for anti-HIF1 $\alpha$ ). Arrowheads indicate hypoxic areas. Scale bar: $1 \mathrm{~mm}$. (E) Quantification of

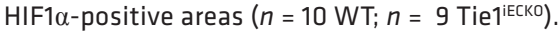
${ }^{* *} P<0.001$, by 2 -tailed Mann-Whitney $U$ test. Error bars represent mean \pm SD. (F) Representative images of necrotic LLC primary tumors (arrowheads indicate H\&E-stained light pink areas). Scale bar: $1 \mathrm{~mm}$. (G) Quantification of necrotic areas from WT and Tie $1^{\mathrm{iECKO}}$ tumors ( $n=10$ mice). Error bars represent mean \pm SD. ${ }^{* *} P<0.001$, by 2 -tailed Mann-Whitney $U$ test. identified limited clinical benefits of cotargeting Ang2 with VEGF in advanced colorectal tumors, which may correspond to earlier preclinical studies suggesting that Ang2 is only transiently active during the early stages of tumor growth $(16,17)$. Whereas Tie2 is well characterized as the primary Ang/Tie signaling receptor, the function of the second Tie receptor, Tie1, is much less understood. Tie1 does not bind the angiopoietin ligands and is to this day considered an orphan receptor (18). Yet, Tie1 is essential for vascular development: Tie1-deficient mice have no overt angiogenetic defects, but their vasculature fails to mature and embryos die during late gestation $(19,20)$. Functionally, Tie1 acts as a contextual regulator of Tie2, as it counterregulates Tie2 in angiogenic tip cells and sustains Tie2 signaling in remodeling stalk cell vasculature (21). As a result, conditional postnatal deletion of Tie1 in ECs results in reduced retinal vascularization, with increased EC apoptosis and induced vascular regression $(21,22)$. In adult mice, the Tie1 ectodomain is cleaved during inflammation, leading to reduced Tie2 phosphorylation and downregulated Tie2 expression in an Ang2-dependent manner. Tie1 shedding thus contributes to the antagonist role of Ang2 on Tie2 signaling $(23,24)$.

Tie1 is functionally involved in important vascular pathologies, including atherosclerosis and tumor angiogenesis $(22,25)$. It has long been known that Tie 1 is prominently upregulated in the intratumoral vasculature (26-30), but its functional contribution to tumor progression has only recently been explored. Condition- al EC Tie1 deletion in adult mice was shown to inhibit angiogenesis, resulting in reduced tumor growth. This is associated with increased Notch pathway activity and results in improved angiopoietin antagonist therapy (22). Building on this seminal work, the present study was aimed at mechanistically dissecting the role of Tie1 during primary tumor growth and elucidating its contribution to metastatic progression.

\section{Results}

Endothelial Tie1 deletion inhibits tumor angiogenesis and delays late tumor growth. To study its role during tumor growth, Tie1 was deleted conditionally in ECs by tamoxifen administration to Tie $1^{f / f l}$ VE-cadherin-Cre ${ }^{\mathrm{ERT} 2}$ mice (referred to hereafter as Tie ${ }^{\mathrm{iECKO}}$ mice), and subsequent growth of Lewis lung carcinoma (LLC) was comparatively analyzed in Tie ${ }^{1 \mathrm{ECKO}}$ and WT littermate mice following s.c. injection of LLC cells. LLC growth was traced for 18 days after tumor cell injection, when mice were sacrificed and the efficacy of Tie1 deletion was validated by quantitative real-time PCR (qRT-PCR) from whole-tumor lysates (Supplemental Figure 1A; supplemental material available online with this article; https:// doi.org/10.1172/JCI94674DS1). Mice with less than 60\% deletion were excluded from further analysis. Tumor growth in WT and Tie $1^{\mathrm{iECKO}}$ mice did not differ until tumors had grown to almost 1 $\mathrm{cm}^{3}$ in size (Figure 1A). Thereafter, growth curves diverged, leading to a significant reduction of tumor growth (Figure 1A). To study the consequences of EC Tie1 deletion on intratumoral vas- 
A

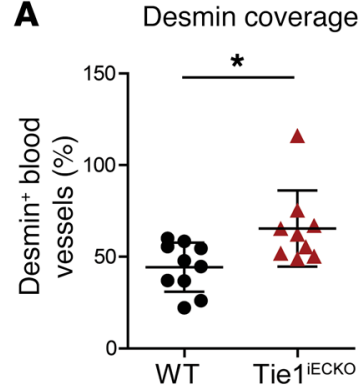

C

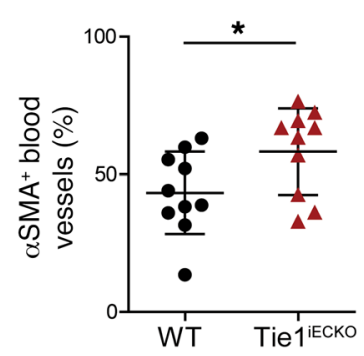

B

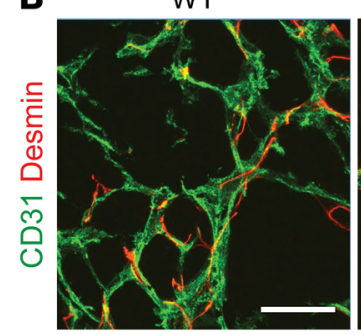

Tie1 $1^{\mathrm{iECKO}}$

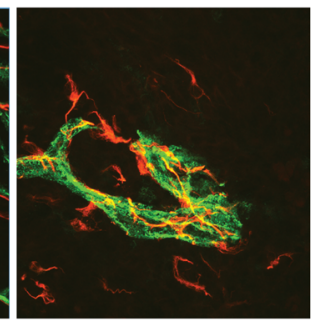

D

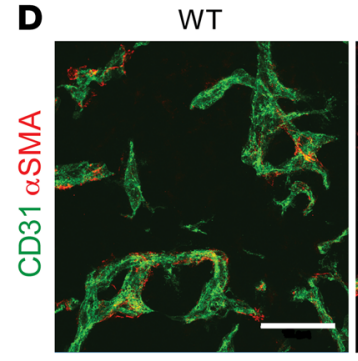

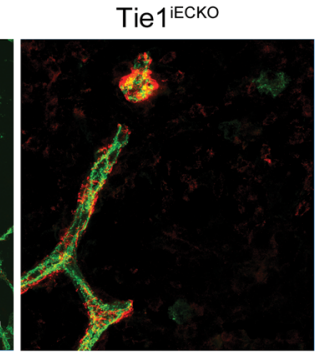

E

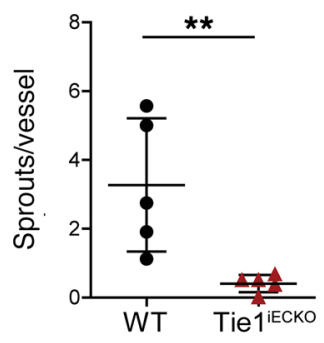

\section{$\mathbf{F}$}

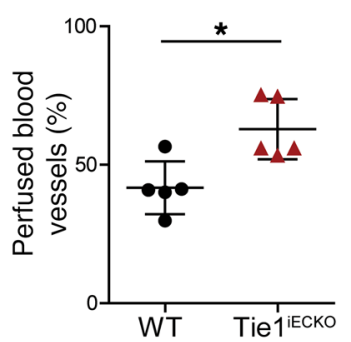

G

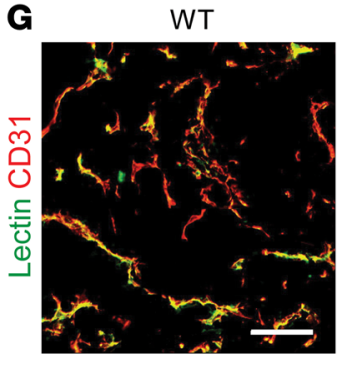

Tie $1^{\mathrm{iECKO}}$

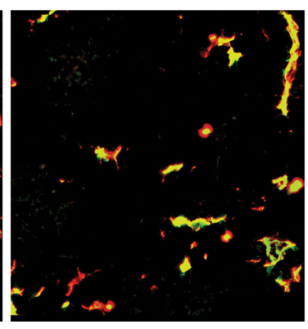

Figure 2. Deletion of endothelial Tie1 leads to increased vascular coverage and improved vessel perfusion. (A) Quantification of desmin-positive blood vessels in WT and Tie1 ${ }^{1 E C K O}$ LLC primary tumors $\left(n=10 \mathrm{WT} ; n=9\right.$ Tie1 $\left.{ }^{\text {ECKO }}\right)$. ${ }^{*} P<0.05$, by 2 -tailed Mann-Whitney $U$ test. (B) Representative confocal images of blood vessels (stained with anti-CD31) and pericytes (stained with anti-desmin). Scale bar: $50 \mu \mathrm{m}$. (C) Quantification of $\alpha$ SMA-positive blood vessels in LLC tumor vasculature $(n=10) .{ }^{*} P<0.05$, by 2-tailed Mann-Whitney $U$ test. (D) Representative microscopic images of blood vessels (stained for anti-CD31) and smooth muscle cells (stained for anti- $\alpha$ SMA). Scale bar: $50 \mu \mathrm{m}$. (E) Quantification of vessel sprouting $(n=5)$. ${ }^{*} P<0.01$, by 2-tailed Mann-Whitney $U$ test. (F) Quantification of lectin-perfused vessel areas $(n=5)$. ${ }^{*} P<0.05$, by 2-tailed Mann-Whitney $U$ test. (G) Representative microscopic images of blood vessels (stained for anti-CD31) perfused with lectin from WT and Tie1 ${ }^{\mathrm{iECK}}$ tumors. Images are representative of 5 mice. Scale bar: $100 \mu \mathrm{m}$. Error bars represent mean \pm SD.

cularization prior to overt tumor growth divergence, we harvested tumors on day 14 and analyzed the microvessel area and density as well as tumor cell hypoxia and necrosis (see Supplemental Figure 1B for deletion efficiency). CD31 vessel staining revealed a strong decrease in microvessel area as well as density in the tumors grown in Tie $1^{\mathrm{i} \text { ECKO }}$ mice (Figure 1, B and C, and Supplemental Figure 2A). Reduced vessel density following endothelial Tie1 deletion resulted in larger areas of hypoxia (Figure 1, D and E, and Supplemental Figure 2, B and C) and significant tumor cell necrosis (Figure $1, F$ and $G$ ). To validate these findings in a second tumor model, we inoculated WT and Tie1 ${ }^{\mathrm{BECKO}}$ mice s.c. with B16F10 melanoma cells. As with the LLC model, the B16F10 cell growth curves between WT and Tie $1^{\mathrm{iECKO}}$ mice progressed simultaneously until tumors had reached $1 \mathrm{~cm}^{3}$ in size (Supplemental Figure 3A). Thereafter, Tie $1^{\mathrm{iECKO}}$ mice showed a significant reduction in tumor growth (Supplemental Figure 3A). Furthermore, we observed a strong antiangiogenic phenotype in Tie ${ }^{\text {ECKO }}$ mice with enhanced intratumoral necrosis after 14 days (Supplemental Figure 3, B-E). Together, the data revealed a substantial antiangiogenic effect of Tie $1^{\mathrm{IECKO}}$ that resulted in a delay in tumor growth, but only at later stages of tumor growth.

Tie ${ }^{\text {IECKO }}$ promotes vascular normalization. We next assessed the vasculature properties of tumors grown in Tie $1^{\mathrm{iECKO}}$ mice for 14 days by quantitating the perivascular coverage and vessel perfusion. We found that mural cell coverage, assessed on the basis of costaining for desmin or aSMA with CD31, was significantly increased in Tie1 $1^{\mathrm{iECKO}}$ tumors compared with that seen in WT tumors (Figure 2, A-D). Moreover, we observed a strong reduction in the number of angiogenic sprouts per vessel (Figure $2 \mathrm{E}$ and Supplemental Figure 5, A and D), indicative of a more mature and quiescent vasculature. We also observed an increase in perivascular coverage in the B16F10 tumor model (Supplemental Figure $4, \mathrm{~A}-\mathrm{D})$. Reduced angiogenic sprouting and increased mural cell coverage resulted in a significant enhancement of perfusion of the 
A

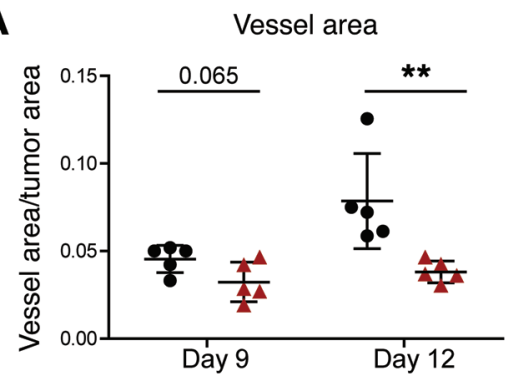

C

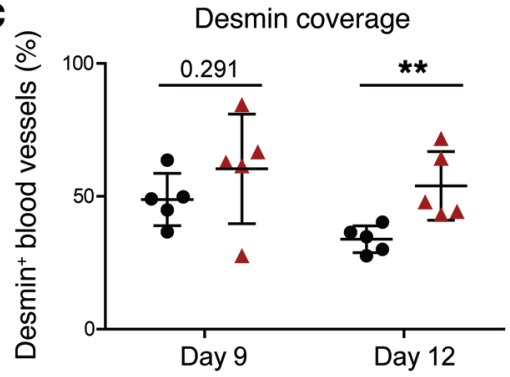

E

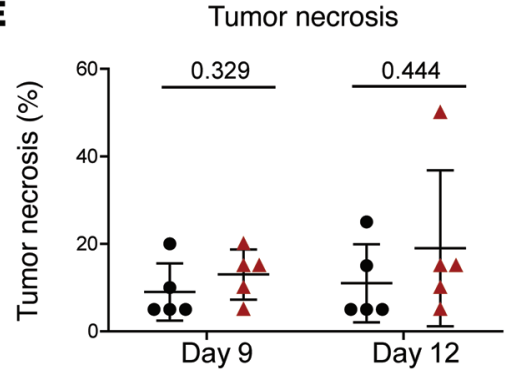

B

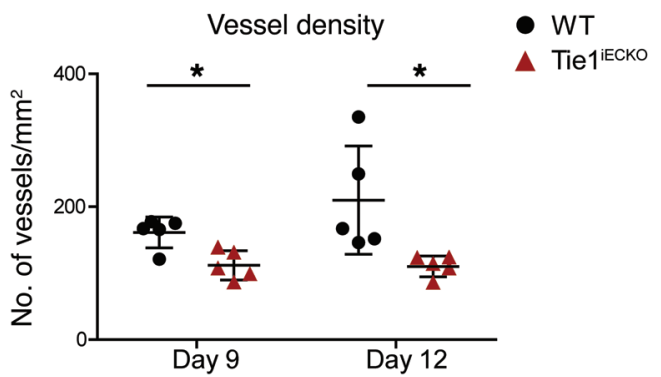

D

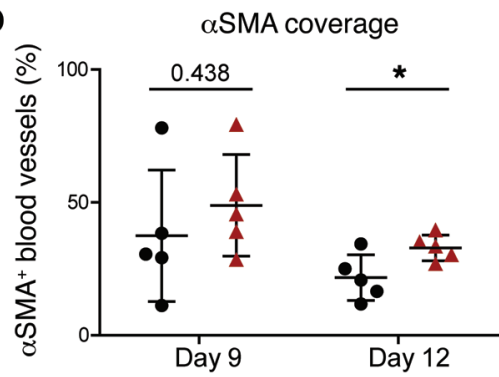

$\mathbf{F}$

Vessel functionality

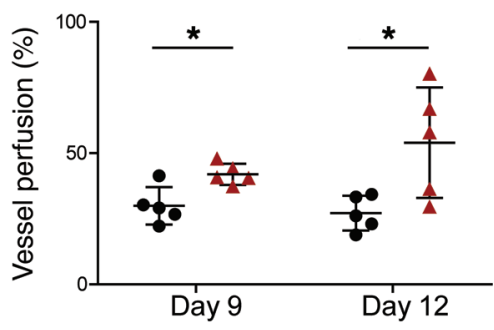

Figure 3. The intratumoral vasculature

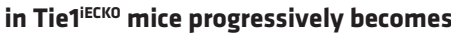
antiangiogenic and normalizes over time during primary tumor growth. (A-D) Quantification of vessel area (A), vessel density (B), and desmin-positive (C) and $\alpha$ SMA-positive (D) blood vessels 9 and 12 days after inoculation with LLC cells $(n=5)$. ${ }^{* *} P<0.01$ and ${ }^{*} P<0.05$, by 2-tailed Mann-Whitney $U$ test. (E and F) Quantification of primary tumor necrosis 9 and 12 days after inoculation of LLC cells $(n=5)(\mathbf{E})$ and analysis of the lectin-perfused vessel area $(n=5)(\mathbf{F})$. ${ }^{*} P<0.05$, by 2 -tailed Mann-Whitney $U$ test. Error bars represent mean \pm SD. remaining intratumoral vessels in Tie $1^{\mathrm{iECKO}}$ mice, evidenced by the intraluminal accumulation of fluorescent lectin injected i.v. into the tumor vasculature (Figure 2, F and G). These findings demonstrated the strong antiangiogenic and provascular normalization effects of Tie1 deletion in ECs, which caused a delay in tumor growth at late stages of tumorigenesis, once the reduced microvessel density became rate limiting.

Endothelial Tie1 deletion induces vessel normalization prior to necrosis progression. To precisely study the role of Tie1 in determining the temporal relationship between angiogenesis, vascular abnormalization, and tumor growth, we performed timeline experiments to analyze the vascular phenotype at earlier stages (days 9 and 12 after tumor cell inoculation). On day 9, we observed no major changes in microvessel area or density (Figure 3, A and B, and Supplemental Figure 6, A and B) or in tip cell sprouting (Supplemental Figure 5, A and B) in Tie ${ }^{\mathrm{E} \mathrm{ECKO}}$ tumors compared with WT tumors. Similarly, we found no differences in desmin- or aSMA-covered vessels (Figure 3, C and D, and Supplemental Figure 6, A and B) or in tumor cell necrosis (Figure $3 \mathrm{E}$ and Supplemental Figure 6D) between the 2 experimental groups. However, there was already a minor but significant improve-

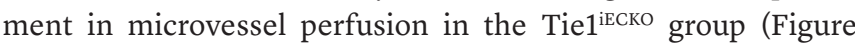
$3 \mathrm{~F}$ and Supplemental Figure 6C). Hence, Tie1 deletion did not drastically affect the early phases of tumor growth but did affect the primary tumor vasculature and, subsequently, tumor growth only at later stages. Correspondingly, the vessel area and density in tumors harvested on day 12 were significantly decreased in

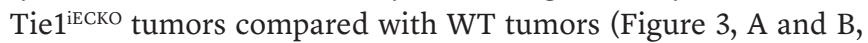
and Supplemental Figure 6, E and F), as was the number of tip cell sprouts per vessel (Supplemental Figure 5, A and C). Concomitantly, mural cell coverage and perfusion were increased in

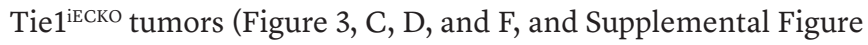
$6, E-G)$, whereas no increase in tumor cell necrosis was detectable in Tie ${ }^{\mathrm{iECKO}}$ tumors on day 12 (Figure $3 \mathrm{E}$ and Supplemental Figure $6 \mathrm{H}$ ). Together, the data indicate that EC Tie1 deletion has a direct effect on the vascularization of primary tumors prior to the build-up of tumor necrosis.

Tie1 promotes tumor cell intravasation, resulting in lung metastasis and reduced survival. Normalized vasculature is more efficient in oxygen and nutrient delivery compared with tumor vasculature and constitutes a tighter endothelial barrier, rendering tumor cells less prone to escape. Normalization is therefore generally associated with a reduction of metastasis (2). To analyze the role of Tie1 during metastasis, we performed tumor resection experiments, in which the primary tumor was surgically removed once it had grown to approximately $0.8 \mathrm{~cm}^{3}$ in size (day 14). This experimental approach circumvented termination of the experiment because of primary tumor growth and also modeled the clinical situation in which tumor patients survive the primary tumor as a result of successful surgery but succumb to metastatic disease that develops after the operation. 
A

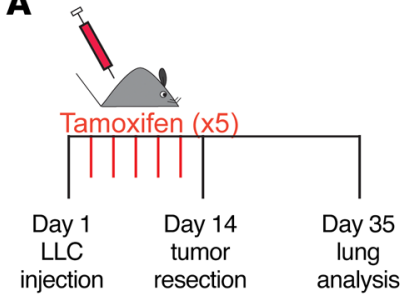

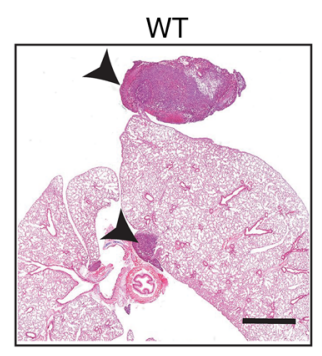

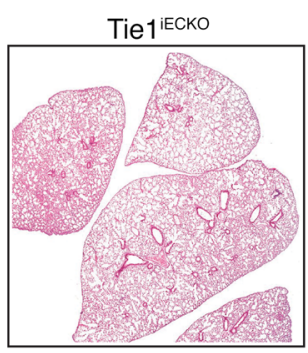

B

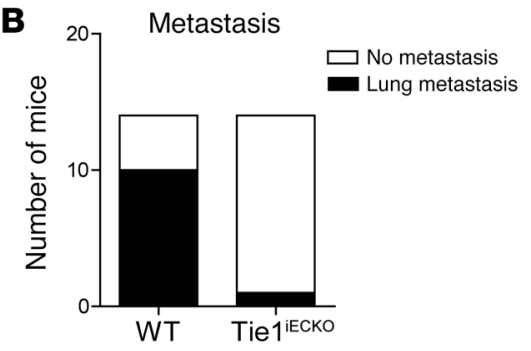

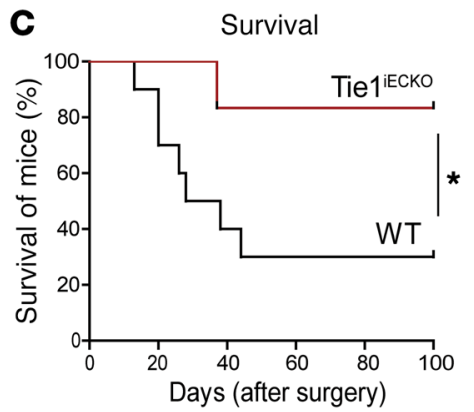

D

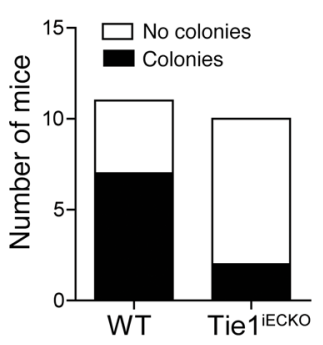

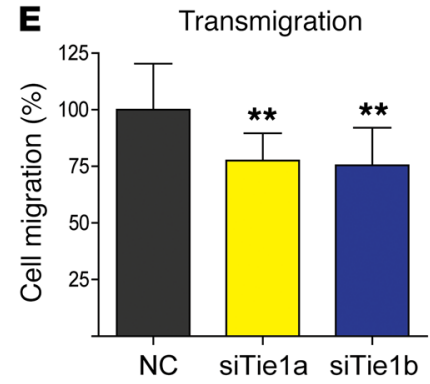

G

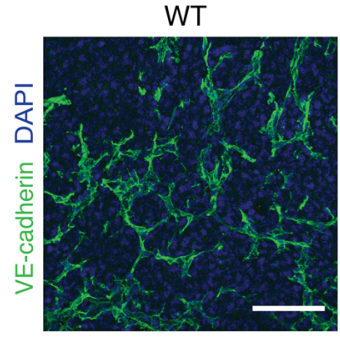

Tie1 $1^{\mathrm{iECKO}}$

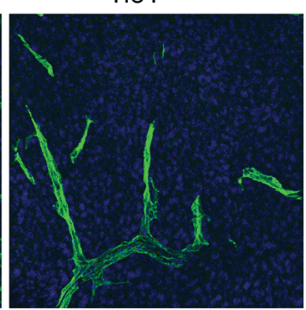

H

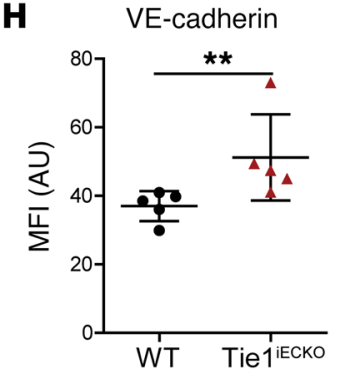

Figure 4. Deletion of endothelial Tie1 reduces tumor cell intravasation and limits metastasis. (A) Schematic representation of the experimental design. LLC cells were injected s.c., and tamoxifen was administrated as indicated. Tumors were surgically removed after 14 days, and mice were sacrificed 3 weeks after primary tumor resection. Shown are representative images of H\&E-stained lungs from WT and Tie ${ }^{\text {iEcko }}$ mice. Arrowheads indicate metastases. Scale bar: $3 \mathrm{~mm}$. (B) Number of mice that developed lung metastases (black) after primary tumor removal $(n=14)$. (C) Kaplan-Meier survival curve of WT and Tie1 ${ }^{\text {IECKO }}$ mice after primary tumor removal ( $n=10 \mathrm{WT} ; n=6$ Tie1 $\left.{ }^{\mathrm{IECKO}}\right)$. ${ }^{*} P<0.05$, by Gehan-Breslow-Wilcoxon test. Mice with less than $50 \%$ endothelial Tie1 deletion were excluded from the analysis. (D) Blood was drawn from WT and Tie1 ${ }^{\text {iEcKo }}$ tumor-bearing mice at the time of tumor removal and plated in culture dishes. The formation of tumor cell colonies was traced over time. Graph shows the number of blood samples that developed tumor cell colonies

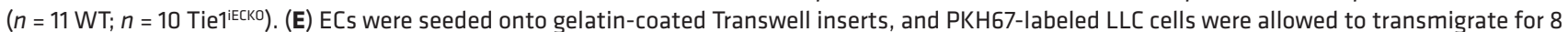
hours. Thereafter, the cells were counted (percentage of LLC cells transmigrated through siTie1-KD HUVECs vs. control; $n=3$ independent experiments, with each experiment performed in triplicate). ${ }^{* *} P<0.01$, by 2-tailed Mann-Whitney $U$ test. (F) Relative VE-cadherin (Cdh5) expression in isolated tumor

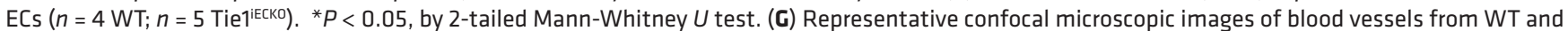
Tie1 $1^{\text {IECKO }}$ tumors stained with VE-cadherin and DAPI. Scale bar: $100 \mu \mathrm{m}$. (H) Quantification of VE-cadherin mean fluorescence intensity $(\mathrm{MFI})(n=5)$.

${ }^{* *} P<0.01$, by 2 -tailed Mann-Whitney $U$ test. Error bars represent mean \pm SD.

WT and Tie $1^{\mathrm{iECKO}}$ mice, in which LLC tumors had been surgically removed 14 days after tumor cell inoculation, were followed for another 3 weeks and sacrificed on day 35 , when the lungs were examined for metastases (Figure 4A). At this time, 10 of $14(71 \%)$ control mice had metastatic foci, whereas only 1 of 14 (7\%) Tie1 ${ }^{\text {iECKO }}$ mice developed metastases (Figure 4B). This translated into improved overall survival of Tie1 $1^{\mathrm{iECKO}}$ mice in another experiment, in which mice were followed for 100 days (Figure 4C). Indeed, $85 \%$ of the animals from the Tie $1^{\mathrm{i} C \mathrm{CKO}}$ group were still alive 100 days after surgery, whereas only $30 \%$ of the control mice had survived (Figure $4 \mathrm{C}$ ).

In order to relate metastasis to the occurrence of circulating tumor cells (CTCs), we next plated in culture freshly drawn blood from tumor-bearing mice at the time of surgical tumor resection. After 2 weeks in culture, we counted outgrown fluorescence-labeled LLC CTC colonies under a fluorescence microscope and found that 7 of 11 cultures obtained from WT mice developed colonies. In contrast, only 2 of 10 cultures obtained from Tie $1^{\mathrm{iECKO}}$ mice grew colonies, suggesting that a reduced number of tumor cells had escaped from the primary tumor site into the circulation upon EC Tie1 deletion (Figure 4D).

In order to study the role of Tie1 in tumor cell transmigration through the endothelium, we performed an in vitro tumor cell transmigration assay, in which fluorescence-labeled LLC cells were allowed to transmigrate through an EC monolayer grown on Transwell inserts. siRNA-mediated silencing of Tie1 in HUVECs resulted in a significant reduction of tumor cell migration across the EC monolayer (Figure 4E). Correspondingly, Cdh5 mRNA in isolated tumor ECs and VE-cadherin protein expression in tumor sections were significantly increased in Tie $1^{\mathrm{ECCKO}}$ mice 14 days after LLC inoculation (Figure 4, F-H). Together, the combination of in vivo and in vitro 
A

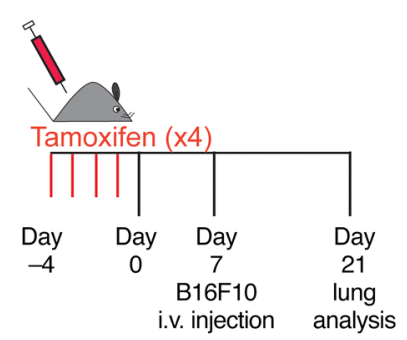

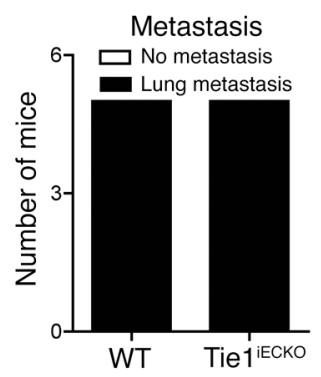

B

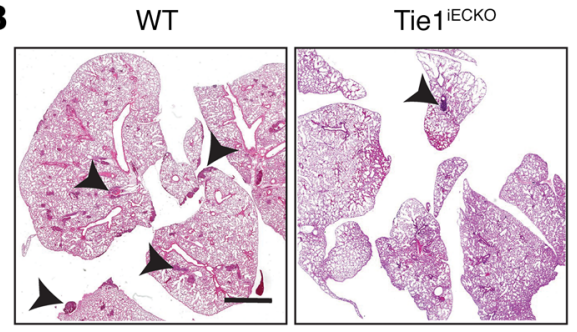

C

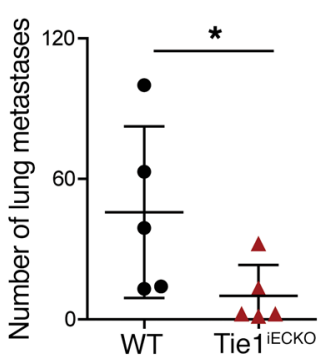

D

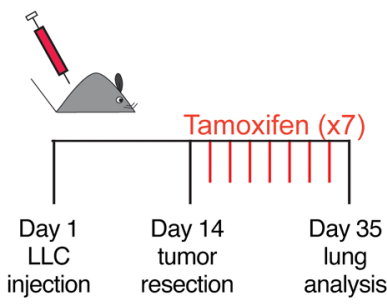

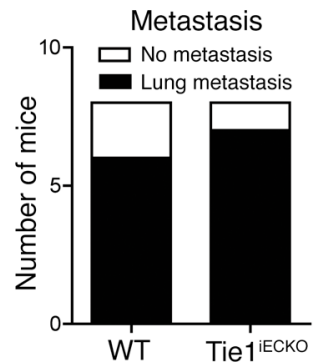

E

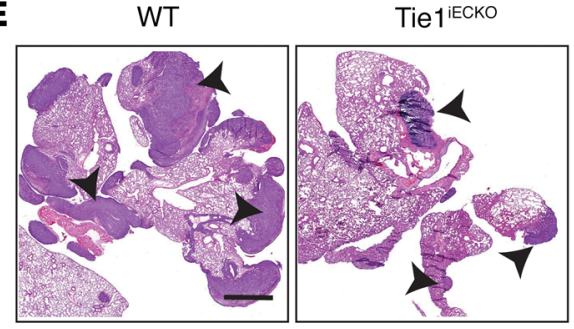

F

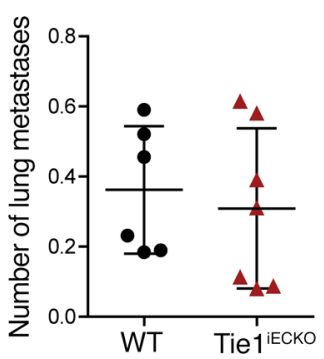

Figure 5. Deletion of endothelial Tie1 reduces tumor cell extravasation and seeding but does not affect seeded micrometastasis. (A) Schematic representation of the experimental metastasis model. Mice were preinjected with 4 doses of tamoxifen, and B16F10 cells were injected i.v. 1 week after the last tamoxifen dose. Mice were sacrificed, and their lungs were analyzed 2 weeks after tumor cell inoculation. The graph shows the number of WT and Tie1 ${ }^{\text {iEcko }}$ mice that developed lung metastases (black) 2 weeks after i.v. injection of B16F10 cells $(n=5)$. (B) Representative H\&E-stained images of lung tissue from WT and Tie1 ${ }^{\text {iECKO }}$ mice. Arrowheads indicate metastatic foci. Scale bar: $3 \mathrm{~mm}$. (C) Quantification of the number of lung metastases per mouse $(n=5)$. ${ }^{*} P<$ 0.05, by 2-tailed Mann-Whitney $U$ test. (D) Schematic representation of the experimental design. LLC cells were injected s.c., and tumors were resected on day 14. Endothelial Tie1 was thereafter deleted via tamoxifen administration. Mice were sacrificed, and lungs were analyzed 3 weeks after the primary tumor resection. Graph shows the number of WT and Tie1 ${ }^{1 E C K O}$ mice that developed lung metastases (black) 3 weeks after surgical removal of the primary tumor $(n=8)$. (E) Representative H\&E-stained images of lung tissue from WT and Tie1 ${ }^{\mathrm{E} C K 0}$ mice. Arrowheads indicate metastases. Scale bar: 3 mm. (F) Quantification of the metastatic areas in lungs from WT and Tie1ЕСКo mice ( $n=6$ WT; $n=7$ Tie1 ${ }^{\text {iECKo }}$ ). Statistical significance was determined by a 2-tailed Mann-Whitney $U$ test. Error bars represent mean \pm SD.

experiments indicated that the reduction in postsurgical lung metastases correlated with a decrease in tumor cell transmigration through the EC layer, leading to a reduction in the number of CTCs. Tie1 thus affects the first steps of the metastatic cascade, whereby tumor cells escape from the primary tumor and intravasate into the circulation.

Tie1 promotes tumor cell extravasation and seeding but does not affect the growth of seeded micrometastases. The previous experiments revealed a metastasis-promoting effect of Tie1 during intravasation. To investigate its contribution to subsequent steps of the metastatic cascade, B16F10 cells were directly i.v. injected into the circulation, allowing the examination of seeding, extravasation, and colonization of CTCs independently of spontaneous intravasation from the primary tumor (31). Mice were injected with 4 doses of tamoxifen (see Supplemental Figure 1C for deletion efficacy), and B16F10 cells were injected via the tail vein 1 week after the last dose of tamoxifen (Figure 5A). We detected metastatic colonies in both WT and Tie $1^{\mathrm{iECKO}}$ mouse lungs, however, Tie $1^{\mathrm{iECKO}}$ mice showed a significant decrease in the number of metastatic foci (Figure 5, B and C), suggesting that Tie1 affected the efficacy of tumor cell seeding and extravasation.

Next, we analyzed whether EC Tie1 deletion would also affect the growth of seeded micrometastases. To this end, we modified the primary tumor tamoxifen application protocol (Figure 4A) to delete Tie1 in lung ECs only after surgical removal of the primary tumor (Figure 5D; see Supplemental Figure 1D for deletion effica- cy). In this experiment, 6 of 8 (75\%) WT mice and 7 of 8 (87.5\%) Tie $1^{\text {iECKO }}$ mice developed macroscopically detectable lung metastases (Figure 5D), with similar lung metastatic areas on histological analysis (Figure 5, E and F). As with the findings in primary tumors, lung metastases in Tie $1^{\mathrm{iECKO}}$ mice showed a decrease in microvessel area and density, but no significant difference in mural cell coverage (Supplemental Figure 7). Taken together, Tie1 deletion inhibited tumor cell extravasation and seeding at secondary sites, but as was observed in the primary tumors, it did not affect the early growth of metastatic tumors.

Tie1 deletion favors Tie2-expressing endothelium and promotes stabilization through increased Ang1 expression. The orphan receptor Tie1 exerts its effects on angiogenesis and vascular maturation by contextually affecting Ang1/Tie2 signaling (21, 23, 24). We therefore performed cytokine array experiments (Supplemental Figure 8A) to assess the protein level of Ang1, Ang2, and other molecules in tumors grown in WT and Tie $1^{\mathrm{iECKO}}$ mice. The expression of both Ang1 and Ang2 was increased in tumors from Tie $1^{\text {iECKO }}$ mice compared with expression levels in WT mouse tumors (Figure 6A and Supplemental Figure 8B). However, the ratio of Ang1 to Ang2 expression shifted toward Ang1 (Figure 6B). Intratumoral expression of VEGF was not significantly affected by EC Tie1 deletion (Supplemental Figure 8, A and C). Interestingly, we detected a strong reduction of $K d r$ (encoding VEGFR2) mRNA expression in FACS-sorted isolated tumor ECs 
A

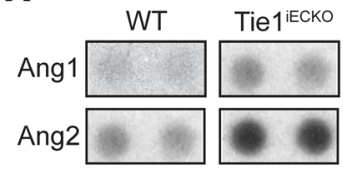

E

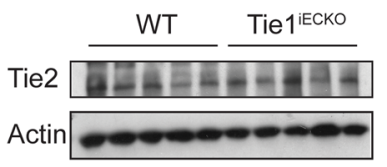

B

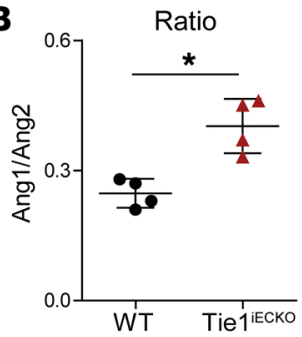

$\mathbf{F}$

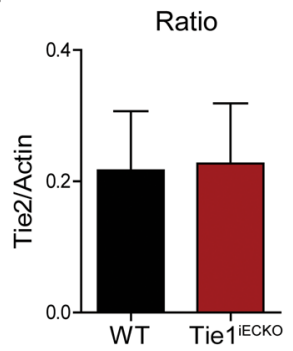

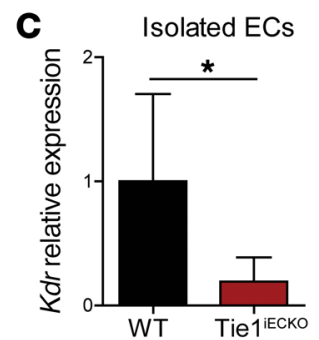

G

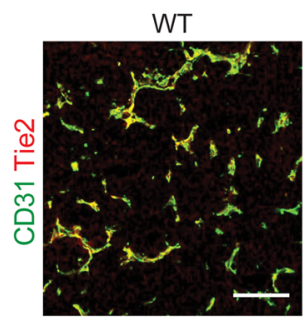

D

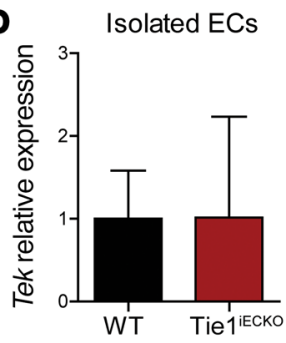

H

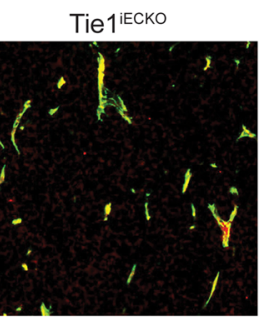

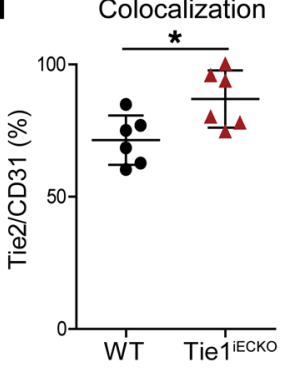

Figure 6. Endothelial Tie1 deletion induces vessel stabilization through Ang1/Tie2 signaling. (A) Representative scans of the cytokine array performed on WT and Tie ${ }^{\text {EECKO }}$ LLC tumor lysates showing Ang1 and Ang2 protein expression $(n=4)$. (B) Quantitation of the cytokine array intensities in A, with quantitation of the Ang1/Ang2 ratio $(n=4)$. ${ }^{*} P<0.05$, by 2-tailed Mann-Whitney $U$ test. (C) Relative $K d r$ mRNA expression in isolated tumor ECs ( $n=4$ WT; $n=5$ Tie $^{1 \text { EECKo }) . ~}{ }^{*} P<0.05$, by 2-tailed Mann-Whitney $U$ test. (D) Relative Tek expression in isolated tumor ECs $\left(n=4\right.$ WT; $n=5$ Tie $\left.{ }^{1 \mathrm{EECKO}}\right)$. (E) Scans of Western blots performed on tumor lysates with Abs against Tie2 and actin as a loading control. Representative images are from 2 independent experiments. (F) Quantitation of the Tie2 and actin blot intensities shown in E ( $n=5$; 2-tailed Mann-Whitney $U$ test). (C) Representative images of CD31-positive tumor blood vessels stained for Tie2. Scale bar: $100 \mu \mathrm{m}$. (H) Quantification of CD31 and Tie2 colocalization $(n=6) .{ }^{*} P<0.05$, by 2-tailed Mann-Whitney U test. Error bars represent mean \pm SD.

from Tie $1^{\text {iECKO }}$ mice, 14 days after tumor cell injection (Figure 6C), confirming that EC $K d r$ expression is strongly regulated by Ang/Tie signaling (32). Unlike Kdr, Tek (encoding Tie2) mRNA levels in FACS-isolated tumor ECs were not altered (Figure 6D), and, similarly, total Tie2 protein levels in tumor lysates were not changed in Tie $1^{\mathrm{iECKO}}$ mice (Figure $6, \mathrm{E}$ and F). However, the microvessel density in Tie $1^{\mathrm{ECCKO}}$ tumors was strongly reduced (Figure 1, C and D), with a pronounced reduction of angiogenic sprouts (Figure 2E). Given that Tie2 is negatively regulated during angiogenesis $(33,34)$, we therefore hypothesized that EC Tie1 may not directly regulate EC Tie2 expression but rather affects the fraction of Tie2-negative angiogenic ECs, as has been suggested during postnatal mouse retinal angiogenesis (21). Indeed, by quantitating the colocalization index of Tie 2 and the pan-endothelial marker CD31 in tumor sections, we detected a significant increase in the percentage of Tie2-positive ECs (stalk cells) in the vasculature of tumors grown in Tie $1^{\mathrm{E} \mathrm{ECKO}}$ mice and, therefore, a reduction of Tie2-negative endothelium (tip cells) (Figure 6, G and H). Quantitative analysis of Tie2 activation in vivo turned out to be technically challenging and did not yield robust or reproducible findings. To circumvent these difficulties, we designed a cellular surrogate experiment, in which cultured HUVECs were exposed to the same Ang1/Ang2 ratio that was observed in the tumors grown in WT and in Tie $1^{\mathrm{iЕCKO}}$ mice (Figure 6B). Cellular mimicry of the knockout (silencing of Tie1 and shift of the Ang1/Ang2 ration from 0.3 to 0.5 ) resulted in a significant increase in phosphorylated Tie2 (p-Tie2) in cultured HUVECs (Supplemental Figure 8, D and E).
In conclusion, the vascular normalization caused by Tie1 deletion in the primary tumor is a result of increased Ang1 in the tumor microenvironment and an increased fraction of Tie2-positive ECs.

\section{Discussion}

In contrast to the Tie2 ligand Ang2, the orphan receptor Tie1 has been much less studied in the context of tumor angiogenesis (22). Like Ang2, Tie1 is transcriptionally strongly upregulated in the angiogenic vasculature and downregulated in resting ECs $(21,26$, $27,29,30,35)$. Functionally, Tie1 has been shown to contextually affect Tie 2 signaling by acting as an antagonist of Tie 2 in angiogenic tip cells and sustaining Tie2 signaling in remodeling stalk cells (21). During inflammation, Tie1 is proteolytically cleaved, which contributes to the regulation of the antagonistic versus agonistic effects of Ang2 on Tie2 $(23,24)$. Building on the previous demonstration of a tumor growth-inhibiting effect of genetic Tie1 deletion (22), we used conditional genetic models of inducible Tie1 deletion at different stages of primary tumor growth and metastasis to show that EC-specific inactivation of Tie1 (a) strongly inhibits sprouting angiogenesis and thereby reduces intratumoral microvessel density and tumor growth; (b) improves perfusion of the residual, more mature, microvessels; (c) limits intravasation of CTCs and thereby reduces metastasis; and (d) limits metastatic tumor cell extravasation.

Conditional deletion of Tie1 in ECs at different stages of primary tumor growth and metastasis confirmed the previously reported antiangiogenic effect of EC Tie1 deletion (22) and enabled beyond that the temporal analysis of Tie 1 effects on tumor 
angiogenesis and metastasis. Indeed, until day 9 of tumor growth, Tie1 deletion did not induce substantial changes in the Tie $1^{\text {iвско }}$ tumor vasculature. Yet, we observed a reduction in tip cell sprouts, resulting in a strong antiangiogenic effect 12 days after tumor cell inoculation that was even more pronounced by day 14 . The progressive and drastic decrease in microvessel area and density in the Tie $1^{\mathrm{i} \text { Еско }}$ tumors led to increased primary tumor hypoxia and subsequent tumor necrosis, which eventually resulted in tumor growth delay only at later stages.

The temporal pattern of Tie1 deletion is in sharp contrast to the effects of genetic Ang2 deletion in the same models, which inhibits early stages of tumor growth but does not affect later stages of tumor progression (17). Although global Ang2 deletion and endothelium-specific Tie1 deletion similarly result in vessel normalization with higher rates of pericyte coverage, the underlying cellular mechanisms appear to be different. Whereas Ang2 deletion affected pericyte coverage with a limited effect on sprouting angiogenesis (17), the increase in vessel normalization in Tie $1^{\mathrm{iECKO}}$ mice appeared to result primarily from the abrogation of the immature tip cell vasculature, leaving behind a more covered and better-perfused mature vasculature. The sustained antiangiogenic effect of EC Tie1 deletion eventually resulted through successive pruning in a normalized (i.e., mural cell covered and well perfused) but, in terms of microvessel density, insufficient, vasculature, leading to hypoxia, necrosis, and tumor growth delay. This interpretation is also supported by Tie ${ }^{\mathrm{iECКO}}$ experiments during physiological postnatal retinal angiogenesis, which caused tip cell apoptosis and vascular regression (21). Likewise, Tie1 deletion has been shown to upregulate the Notch pathway (22), which similarly argues for a primary tip cell phenotype. As such, differences in the target cell population of Ang2 and Tie1 could mechanistically explain the previous observation that, in preclinical models, Tie1 targeting synergizes with blocking of the angiopoietins, but not with targeting of the VEGF/VEGFR axis (22).

In line with the effect of Tie1 deletion on sprouting tip cells, Tie $^{\text {iECKO }}$ led to the strengthening of endothelial adherens junctions by upregulating $C d h 5$ mRNA and protein expression. Tighter endothelial junctions and higher overall perivascular coverage preserve vascular integrity, leading to improved vessel perfusion with decreased vessel permeability. Therefore, Tie $1^{\text {iЕско }}$ blood vessels form a strong barrier that is less prone to tumor cell intravasation at the primary tumor site and extravasation at the metastatic site. This was validated in the present study in a postsurgical spontaneous

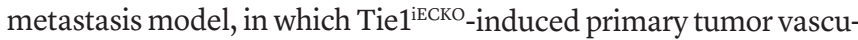
lature normalization exerted an inhibitory effect on lung metastasis, with increased overall survival. Tie1 deletion in this model not only affected early intravasation steps during the metastatic cascade, but also extravasation and seeding at the secondary site. Yet, Tie1 deletion at later steps, when metastatic colonization had taken place, did not reduce metastatic incidence or burden, but phenocopied the primary tumor behavior with reduced microvessel area and density (the late primary tumor growth delay could not be observed in the metastases, because the experiment had to be abandoned for ethical reasons). Similar findings have recently been reported for neuroblastomas, in which targeting of stathmin limited the number of tumor nodules by reducing tumor cell extravasation and seeding, without affecting the growth of preseeded micrometastases (36).
Mechanistically, the contribution of Tie1 to Tie 2 signaling is still poorly understood. Because Tie1 is not ligand binding, it has been hypothesized that Tie1 contributes to Tie2 signaling by heterodimerization via interaction of the fibronectin type III domain n3 (9) and/or by affecting the binding of the angiopoietin ligands to Tie2 (21, 37-39). Elegant recent work has shown that acute inflammation causes Tie1 ectodomain shedding, which in turn leads to downregulation of Tek mRNA, reduction of p-Tie2, and upregulation of Ang2 expression. In this context, Tie1 shedding prevents the agonistic action of Ang2 and favors its antagonistic role on Tie2, thereby leading to vascular remodeling $(23,24)$. Similar effects were observed in Tie1 ${ }^{\mathrm{iECKO}}$ mice, in which deletion of the orphan receptor favored Ang2 antagonistic activity on Tie2 (23). However, these effects were only observed in acutely challenged mice. As such, the effects in chronically growing tumors may be different from those seen with acute inflammation and sepsis. In fact, we observed in the present study that Tie1 deletion did not induce changes in Tie 2 mRNA expression, but promoted an increase in Tie 2 protein on a per-EC basis and an enhancement of its phosphorylation levels (studied in a cellular surrogate experiment). Moreover, both, Ang1 and Ang2 were upregulated, with the Ang1/Ang2 ratio being shifted toward Ang1. Ang1 overexpression is known to increase Tie2 activation and maintain a quiescent EC phenotype while sustaining vessel integrity $(40,41)$. Furthermore, endothelial $K d r$ expression was strongly downregulated, which corresponded to the reported regulation of $K d r$ by Ang2 during liver regeneration (32). Together, the concordance of the gene expression changes strongly argues for a Tie2 gain-of-function phenotype, with antiangiogenic- and vessel normalization-promoting effects in the tumor vasculature of Tie $1^{\text {iЕско }}$ mice. Corresponding to these results, the ligand-independent activation of Tie2 through pharmacological inhibition of VE-PTP has been shown to result in normalization and enhanced perfusion of the primary tumor vasculature. Yet, as in the present study, this was an early tumor growth phenotype that was associated with a reduction of tumor cell extravasation at secondary sites and delayed progression of existing micrometastases (42). An inhibition of tumor growth and of distant metastasis has also been reported by blocking of the Ang ligands through adenoviral administration of soluble Tie2 (43). The authors had interpreted this as a Tie2 inhibition phenotype. However, shift of the Ang1/Ang2 ratio toward Ang2 within tumors more likely suggests that the resulting phenotype actually occurred as a consequence of Tie2 gain of function (43).

Antiangiogenic therapies have thus far primarily focused on the VEGF/VEGFR axis. VEGF is the predominant angiogenic factor produced by cancer and stromal cells during tumor neovascularization (44). However, this strategy has shown limitations, mainly because of refractoriness or evasive escape of the tumor cells (45). To overcome these limitations, second-generation antiangiogenic drugs have been developed for combination therapies with established anti-VEGF/VEGFR therapies. In this context, the strong upregulation of Ang2 in the tumor endothelium $(46,47)$ and its destabilizing effect on tumor vasculature (48) have made this cytokine a very attractive therapeutic target. Its inhibition in association with VEGF therapy normalizes tumor vasculature and has shown pronounced effects on overall survival in preclinical tumor models $(49,50)$. Yet, these preclinical findings have not yet translated into an improved therapeutic effect of VEGF/ 
Ang2 cotargeting in human clinical trials (16). The present study provides experimental evidence suggesting that Tie1 targeting is mechanistically and temporally different from Ang2 and VE-PTP blockade and supports a strong rationale for exploiting Tie1 as a therapeutic target. Tie1 inhibition strongly promotes intratumoral vascular normalization with downregulation of VEGFR2 and a shift of the Ang1/Ang2 ratio toward Ang1. It thereby exerts antiangiogenic and antimetastatic effects. Moreover, the strong differential of Tie1 expression in the angiogenic versus the resting vasculature makes Tie1 an attractive target for therapeutic applications. Future work will need to establish under what conditions Tie1 blockade acts synergistically with anti-VEGF/VEGFR therapy or whether Tie1 targeting could potentiate Ang2-targeting drugs in triple Tie1/Ang2/VEGF combination therapies.

\section{Methods}

Cells. HUVECs (obtained from Promocell) were cultured in endopan-3 medium completed with the corresponding supplements (PAN Biotech $\mathrm{GmbH}$ ). LLC cells (obtained from ATCC), LLC-red fluorescent protein (LLC-RFP) cells (provided by Andreas Fischer, DKFZ), and B16F10 cells (ATCC) were cultured in DMEM plus 10\% FCS (Invitrogen, Thermo Fisher Scientific) and 1\% penicillin-streptomycin. All cells were cultured at $37^{\circ} \mathrm{C}$ and $5 \% \mathrm{CO}_{2}$ and routinely tested negative for mycoplasma by PCR.

Transfection of cells. For gene-silencing experiments, HUVECs were separately transfected with $100 \mathrm{nM}$ of 2 different Tie1 siRNAs (s14140 and s14142) or noncoding siRNA (Life Technologies, Thermo Fisher Scientific) using Oligofectamine (Life Technologies, Thermo Fisher Scientific; 12252-011) in Opti-MEM plus GlutaMAX-I (Life Technologies, Thermo Fisher Scientific). Validation of Tie1 downregulation was done by qRT-PCR, and functional experiments were performed 48 hours after transfection, when the deletion efficacy was $80 \%$ and $70 \%$ for $\mathrm{s} 14140$ and s14142, respectively.

HUVEC stimulation assay. A surrogate cellular experiment was designed to study the consequences of Tie1 deletion in combination with a shift of the Ang1/Ang2 ratio as observed in the in vivo tumor experiments (Figure 6B). To mimic the WT condition, HUVECs were transfected with control siRNA and stimulated with a combination of $200 \mathrm{ng} / \mathrm{ml}$ recombinant Ang1 (R\&D Systems) and $650 \mathrm{ng} / \mathrm{ml}$ recombinant Ang2 (R\&D Systems). To mimic the Tie $1^{\text {iЕСКо }}$ condition, HUVECs were transfected with Tie1 siRNA (2 independent siRNAs) and stimulated with a combination of $400 \mathrm{ng} / \mathrm{ml}$ recombinant Ang1 and $800 \mathrm{ng} / \mathrm{ml}$ recombinant Ang2 (different ratio and difference in absolute concentrations reflecting the findings of the intratumoral analyses; Supplemental Figure 8B). Prior to stimulation, HUVECs were starved for 6 hours in basal medium (Endopan). EC stimulation was performed in EC basal medium for 20 minutes. Cells were then washed with cold $1 \times$ PBS and lysed for human p-RTK array analysis.

Transmigration assay. Control-transfected or Tie1-silenced HUVECs $\left(1 \times 10^{5}\right)$ were plated in Endopan medium in the top chamber of $6.5-\mathrm{mm} / 8.0-\mu \mathrm{m} 0.2 \%$ gelatin-coated Transwells (Corning) overnight. PKH67 red fluorescent dye-labeled (MilliporeSigma) LLC cells $\left(1 \times 10^{5}\right)$ were added to the top chamber in DMEM $10 \%$ FCS, with DMEM 10\% FCS in the bottom chamber. Eight hours later, Transwells were washed and fixed with Roti-Histofix $4 \%$ for ten minutes, and cell numbers were analyzed by fluorescence microscopy and quantified using ImageJ software (NIH).
Mice. C57BL/6 Tie $1^{f / f l}$ mice (51) were crossed with C57BL/6 $\mathrm{Tg}\left(\mathrm{Cdh} 5\right.$-cre/ERT2)1Rha (VE-cadherin-Cre $\left.{ }^{\text {ERT2 }}\right)$ mice (52) to specifically delete the Tie1 gene in ECs upon tamoxifen application. Floxed mice, equally treated with tamoxifen, were used as controls (WT) in all experiments. Genotyping of mouse samples was performed by PCR of DNA samples using the following primers: Tie ${ }^{f / / f l}$ forward, ATGCCTGTTCTATTTATTTTTCCAG; Tie ${ }^{f l / f l}$ reverse, TCGGGCGCGTTCAGAGTGGTAT; VE-cadherin-Cre ${ }^{\text {ERT2 }}$ forward, GCCTGCATTACCGGTCGATGCAACGA; and VE-cadherin-Cre ${ }^{\text {ERT2 }}$ reverse, GTGGCAGATGGCGCGGCAACACCATT. Mice were housed in individually ventilated cages under pathogen-free conditions. Animals had free access to food and water and were kept on a 12-hour light/12hour dark cycle.

Tumor experiments. For tumor experiments, 7- to 9-week-old male and female mice were s.c. injected with $10^{6}$ LLC cells or B16F10 melanoma cells. To induce Tie1 deletion after tumor cell implantation, both control and mutant animals were i.p. treated with 5 to 7 doses of tamoxifen (2 mg) (MilliporeSigma) dissolved in ethanol and peanut oil, according to the different schedules indicated in Figure $4 \mathrm{~A}$ and Figure 5D. Mice with less than 60\% Tie1 deletion were excluded from further analysis, unless otherwise stated. LLC and B16F10 tumors were harvested 9,12, 14, or 18 days after cell injection. Tumor volume was calculated by measuring the length times the width times the height. The tumor tissue was embedded in Tissue-Tek OCT compound or snap-frozen in liquid nitrogen for further analysis. When lung metastases were analyzed, LLC primary tumors were surgically removed 14 days after cell injection, and the mice were sacrificed 3 weeks after surgery. The lungs were then harvested and processed for histological analysis. Survival studies were performed until the natural death of the mice or until the mice needed to be sacrificed for ethical reasons. The surviving mice were sacrificed 100 days after surgery.

For tail-vein metastasis experiments, mice were preinjected with 4 tamoxifen doses (as indicated in Figure $5 \mathrm{~A})$. B16F10 cells $\left(1 \times 10^{5}\right)$ were injected i.v. 7 days after the last injection. Lungs were collected after 2 weeks and processed for histological analysis.

In vivo hypoxia assay. In order to study tumor tissue hypoxia, LLC cells were s.c. implanted. Tumors were harvested on day 14 or 18. Hypoxyprobe (Hypoxyprobe; Hypoxyprobe Plus Kit) was i.p. injected 45 minutes prior to sacrifice $(60 \mathrm{mg} / \mathrm{kg}$ in $150 \mu \mathrm{l} \mathrm{NaCl})$. Tumors were harvested in Tissue-Tek OCT and stained according to the manufacturer's protocol.

Ex vivo culture of CTCs. Arterial blood was isolated by cardiac puncture and kept in lithium heparin Microtainer Tubes (BD Biosciences) on ice. Blood (100 $\mu \mathrm{l})$ was plated in $10 \mathrm{ml}$ DMEM plus $10 \%$ FCS and $1 \%$ penicillin-streptomycin in a $10-\mathrm{cm}$ tissue culture plate. Tumor cell colonies were visualized under a fluorescence microscope after 14 days. The total number of tumor cell colonies per plate was quantified manually in 2 or more plates for each animal on an Olympus IX71 microscope.

Immunofluorescence and immunohistochemical staining and analyses. For immunofluorescence staining, primary tumors were embedded in Tissue-Tek OCT compound. Cryosections were cut into 7- or $50-\mu \mathrm{m}$ sections and fixed in ice-cold methanol for 20 minutes at $-20^{\circ} \mathrm{C}$. Sections were then blocked and permeabilized in 10\% ready-to-use normal goat serum (Life Technologies, Thermo Fisher Scientific) for 1 hour or 3 hours at room temperature (RT), followed by incubation with the appropriate primary Abs overnight at $4^{\circ} \mathrm{C}$. Incubation with the sec- 


\section{Table 1. List of Abs used for EC isolation by FACS sorting}

\begin{tabular}{lcc} 
Target protein & Species & Company \\
ms CD45-FITC & Rat & BD Pharmingen \\
ms Ter119-FITC & Rat & BD Pharmingen \\
ms Lyve1-FITC & Rat & eBioscience \\
ms podoplanin-A488 & Hamster & eBioscience \\
ms CD31-APC & Rat & BD Pharmingen \\
ms, mouse. & & \\
\hline
\end{tabular}

ondary Ab was performed for 1 hour or 2 hours at RT. Images were taken using a Zeiss Cell Observer and Zeiss Axio Scan, and image analysis was performed using Fiji Is Just ImageJ (Fiji) software.

To quantify tumor vessel density and area, the vessel numbers and CD31-positive vessel area were measured and normalized to the tumor area. For analysis of mural cell coverage and vessel functionality, the aSMA- and desmin-positive area and the lectin-positive area, respectively, were measured and normalized to the corresponding vessel area. For Hypoxyprobe analysis, the pimonidazole-positive area was quantified and normalized to the tumor area. Tip cells analysis was performed on $50-\mu \mathrm{m}$ sections. Five fields per mouse, acquired with a $\times 40$ objective, were used to manually quantify the sprouts, which were related to the number of vessels. An investigator blinded to the treatment groups performed the analyses on an acquired tile scan of entire tumor sections. Two tumor sections seventy micrometers apart from each other were used to quantify vessel area and density; one section per tumor was used for $\alpha$ SMA, desmin, and pimonidazole quantification.

For immunohistochemical staining, zinc-fixed lungs and primary tumors were paraffin embedded, sectioned, and subsequently stained with $\mathrm{H} \& \mathrm{E}$ at the Institute of Pathology (Heidelberg University). Sections were analyzed blindly by a board-certified pathologist (CM), and the percentage of necrotic area per slide in each tumor was assessed. Images were taken using a Zeiss Axio Scan, and image analysis was done using Fiji software in order to analyze the metastatic area in the lungs. To quantify metastatic burden, the metastatic area was measured and normalized to the total lung area. One lung section per mouse was analyzed.

To stain the hypoxic area with a HIF1 $\alpha$ marker in the primary tumors, paraffin sections were dewaxed and rehydrated through a graded series of ethanol. Antigen unmasking was performed using citrate buffer $\left(0.01 \mathrm{M}\right.$ sodium citrate, $\mathrm{pH}$ 6) in a water bath at $95^{\circ} \mathrm{C}$ for 20 minutes, followed by cooling in distillated $\mathrm{H}_{2} \mathrm{O}$. To block the background activity of endogenous peroxidases, the slides were treated with $3 \% \mathrm{H}_{2} \mathrm{O}_{2}$ for 15 minutes. The slides were incubated with $10 \%$ rabbit serum for 30 minutes at RT and further overnight at $4^{\circ} \mathrm{C}$ with primary Ab. The secondary Ab was incubated for 30 minutes at RT. Detection was performed via a biotin-peroxidase complex using VECTASTAIN ABC solution (Vector Laboratories) according to the manufacturers' protocol. Sections were counterstained with hematoxylin for 2 minutes and then rinsed with running tap water for 10 minutes. To quantify the hypoxic area, the HIF1 $\alpha$-positive area was measured and normalized to the tumor area using Fiji software. The analysis was performed blindly.

Abs. The following primary Abs were used for immunofluorescence: rat anti-CD31 (BD Biosciences; catalog 550300); rat anti-cadherin-5 (BD Biosciences; catalog 550548); rabbit anti-desmin (Abcam; catalog Ab15200-1); mouse anti- $\alpha$ SMA (MilliporeSigma; catalog C6198); and rat anti-Tie2 (eBioscience; catalog 14-5987-81). For pimonidazole staining, the 4.3.11.3 mouse FITC-mAb (Hypoxyprobe; HP21000 Kit) was used. Secondary anti-rat A488, anti-rabbit A546, and anti-rat A546 Abs (Life Technologies, Thermo Fisher Scientific) were used. Nuclei were stained with Hoechst (MilliporeSigma). For immunohistochemical analysis, primary mouse anti-HIF1 $\alpha \mathrm{Ab}$ (Abcam; catalog ab8366) was detected with anti-mouse HRP (Dako Cytomation) secondary Ab. For Western blotting, rabbit anti-Tie2 (Santa Cruz Biotechnology; catalog sc-324) and rabbit anti-actin (Cell Signaling Technology; catalog 4967) Abs were used.

FACS sorting of tumor ECs. Mice with tumors were sacrificed, and tumors were minced into small pieces. Single-cell suspensions were prepared by digesting the tissue in DMEM containing $200 \mathrm{U} /$ $\mathrm{ml}$ collagenase I (MilliporeSigma; C9891) and $10 \mu \mathrm{g} / \mathrm{ml}$ DNase I (MilliporeSigma; D4527) at $37^{\circ} \mathrm{C}$ for 30 minutes. The cell suspension was subsequently sieved through a $100-\mu \mathrm{m}$ cell strainer using the plunger of a 19-gauge cannula syringe. The cell suspension was incubated with $30 \mu \mathrm{l} \mathrm{CD31} \mathrm{microbeads} \mathrm{(Miltenyi} \mathrm{Biotec;} \mathrm{130-097-}$ 418) for 20 minutes at $4^{\circ} \mathrm{C}$ in $200 \mu \mathrm{l}$ PBS containing 5\% FCS. Next, the cells conjugated with CD31 microbeads were magnetically concentrated. The isolated cells were then stained with CD45, PDPN, LYVE1, Ter119, and CD31 Abs (Table 1) in PBS containing 5\% FCS for 20 minutes at $4^{\circ} \mathrm{C}$. Dead cells were excluded by FxCycle Violet staining $(10 \mu \mathrm{g} / \mathrm{ml})$ (Invitrogen, Thermo Fisher Scientific; F10347). CD45-Ter119-LYVE1-PDPN ${ }^{-}$CD $31^{+}$cells were sorted with a BD Biosciences FACSAria Cell Sorter.

RNA isolation and qRT-PCR analysis. Tumors were lysed with TRIzol, and RNA was isolated with an RNeasy Mini Kit (QIAGEN) according to the manufacturer's protocols, and RNA from HUVECs was similarly isolated with an RNeasy Mini Kit. The concentration and purity of RNA were measured with the RNA program of the Qubit 2.0 Fluorometer (Invitrogen, Thermo Fisher Scientific). mRNA was transcribed into cDNA using a QuantiTect Reverse Transcription Kit or a QuantiTect Whole Transcriptome Kit (both from QIAGEN) according to the manufacturer's instructions.

RNA from FACS-sorted mouse ECs was isolated with an Arcturus PicoPure RNA Isolation Kit (Life Technologies, Thermo Fisher Scientific), and cDNA was amplified with a QuantiTect Whole Transcriptome Kit.

Subsequent qRT-PCR was performed using a TaqMan gene expression assay (Table 2), TaqMan Fast Advanced Master Mix, TaqMan probes, and Applied Biosystems StepOnePlus (all from Thermo Fisher Scientific). Gene expression relative to the appropriate housekeeping gene was calculated by the $\Delta \Delta \mathrm{Ct}$ method.

\section{Table 2. List of TaqMan assays}

$\begin{array}{llc}\text { Target gene } & \text { Species } & \text { Assay ID } \\ \text { Actb } & \text { Mouse } & \text { Mm00607939-s1 } \\ \text { Cdh5 } & \text { Mouse } & \text { Mm03053719-s1 } \\ \text { Pecam1 } & \text { Mouse } & \text { Mm01242584_m1 } \\ \text { Tie1 } & \text { Mouse } & \text { Mm00441786_m1 } \\ \text { Tek } & \text { Mouse } & \text { Mm00443254_m1 } \\ \text { Vegfr2 } & \text { Mouse } & \text { Mm01222421_m1 }\end{array}$


Western blot analysis. Tumor pieces were lysed in a Tris-NaCl buffer $(25 \mathrm{mM} / 150 \mathrm{mM})$ containing $5 \mathrm{mM}$ EDTA, 1\% NP-40, $100 \mathrm{mM}$ $\mathrm{NaF}$, and $10 \mathrm{mM} \mathrm{Na} \mathrm{P}_{2} \mathrm{O}_{7}$ in the presence of $1 \mathrm{mM}$ orthovanadate and Proteinase-Inhibitor Mix G (SERVA Electrophoresis). The tumor lysates were heated to $95^{\circ} \mathrm{C}$ in sample buffer, subjected to SDS-PAGE, and blotted onto a $\mathrm{MeOH}$-activated PVDF membrane probed with the desired Abs. Western blot analysis was performed using HRP-conjugated secondary Abs (DAKO), and bound $\mathrm{Ab}$ was visualized using ECL (Pierce, Thermo Fisher Scientific). When indicated, the resultant intensities were quantified using Fiji software.

Proteome profiler. The cytokine array was performed on tumor pieces lysed in 1\% Triton X-100 buffer in TBS-T in the presence of $1 \mathrm{mM}$ orthovanadate according to the manufacturer's instructions (R\&D Systems). Four samples (50 $\mu$ g each) per condition were used, and each dot blot was quantified using Fiji software after background subtraction.

A human p-RTK array was performed on HUVECs lysed in 10 $\mu \mathrm{g} / \mathrm{ml}$ aprotinin (MilliporeSigma), $10 \mu \mathrm{g} / \mathrm{ml}$ leupeptin (Tocris), and 10 $\mu \mathrm{g} / \mathrm{ml}$ pepstatin (Tocris) in Lysis Buffer 17 (R\&D System) according to the manufacturers' instructions. A total amount of $150 \mu \mathrm{g}$ protein, pooled from 4 independent experiments of the same siRNA transfection (37.5 $\mu$ g each), was used. Quantification was performed using Fiji software after background subtraction.

Statistics. Statistical analysis was performed using GraphPad Prism version 5 (GraphPad Software). Data are expressed as the mean \pm SD or SEM. Comparisons between 2 groups were made using a 2-tailed Student's $t$ test, a Mann-Whitney $U$ test, 2-way ANOVA, or a Gehan-Breslow-Wilcoxon test. A P value of less than 0.05 was considered statistically significant.

Study approval. All animal experiments were approved by the Bezirksregierung Karlsruhe regulatory committee in Karlsruhe, Germany (permits G61/13 and G171/15).

\section{Author contributions}

SLP, LR, SS, and HGA conceived and supervised the research, analyzed data, and wrote the manuscript. SLP, LR, SS, MS, CS, BS, and $\mathrm{CM}$ performed experiments and analyzed data. All authors read and approved the submission of this manuscript.

\section{Acknowledgments}

The authors would like to acknowledge the excellent technical support of the DKFZ laboratory animal facility and the DKFZ light microscopy core facilities. We wish to thank Anja Runge (German Cancer Research Center, Heidelberg, Germany) for valuable suggestions and discussions. This work was supported by the SFBTR23 Vascular Differentiation and Remodeling project (project A3, to HGA); the SFB873 Maintenance and Differentiation of Stem Cells in Development and Disease project (project B6, to HGA); and the Leducq Transatlantic Network of Excellence - Lymph Vessels in Obesity and Cardiovascular Disease (to HGA).

Address correspondence to: Hellmut G. Augustin, Joint Research Division of Vascular Biology, Medical Faculty Mannheim (CBTM), Heidelberg University, German Cancer Research Center (DKFZ-ZMBH Alliance), Im Neuenheimer Feld 280, 69221 Heidelberg, Germany. Phone: 49.6221.421500; Email: augustin@angiogenese.de.

SS's present address is: Department of Cell and Molecular Biology, Karolinska Institute, Stockholm, Sweden.

SLP, LR, MS, and HGA's present address is: Department of Vascular Biology and Tumor Angiogenesis, European Center for Angioscience (ECAS), Medical Faculty Mannheim, Heidelberg University, Mannheim, Germany.
1. Jain RK. Normalizing tumor microenvironment to treat cancer: bench to bedside to biomarkers. J Clin Oncol. 2013;31(17):2205-2218.

2. Jain RK. Normalization of tumor vasculature: an emerging concept in antiangiogenic therapy. Science. 2005;307(5706):58-62.

3. Baluk P, Hashizume H, McDonald DM. Cellular abnormalities of blood vessels as targets in cancer. Curr Opin Genet Dev. 2005;15(1):102-111.

4. Nagy JA, Chang SH, Shih SC, Dvorak AM, Dvorak HF. Heterogeneity of the tumor vasculature. Semin Thromb Hemost. 2010;36(3):321-331.

5. Ivy SP, Wick JY, Kaufman BM. An overview of small-molecule inhibitors of VEGFR signaling. Nat Rev Clin Oncol. 2009;6(10):569-579.

6. Jain RK. Antiangiogenesis strategies revisited: from starving tumors to alleviating hypoxia. Cancer Cell. 2014;26(5):605-622.

7. Augustin HG, Koh GY, Thurston G, Alitalo K. Control of vascular morphogenesis and homeostasis through the angiopoietin-Tie system. Nat Rev Mol Cell Biol. 2009;10(3):165-177.

8. Davis $\mathrm{S}$, et al. Isolation of angiopoietin-1, a ligand for the TIE2 receptor, by secretion-trap expression cloning. Cell. 1996;87(7):1161-1169.

9. Leppänen VM, Saharinen P, Alitalo K. Structural basis of Tie2 activation and Tie2/Tie1 heterodimerization. Proc Natl Acad Sci U S A. 2017;114(17):4376-4381.
10. Daly C, et al. Angiopoietin-2 functions as a Tie2 agonist in tumor models, where it limits the effects of VEGF inhibition. Cancer Res. 2013;73(1):108-118.

11. Yuan HT, Khankin EV, Karumanchi SA, Parikh SM. Angiopoietin 2 is a partial agonist/antagonist of Tie2 signaling in the endothelium. Mol Cell Biol. 2009;29(8):2011-2022.

12. Lobov IB, Brooks PC, Lang RA. Angiopoietin-2 displays VEGF-dependent modulation of capillary structure and endothelial cell survival in vivo. Proc Natl Acad Sci USA . 2002;99(17):11205-11210.

13. Holopainen T, et al. Effects of angiopoietin2-blocking antibody on endothelial cell-cell junctions and lung metastasis. J Natl Cancer Inst. 2012;104(6):461-475.

14. Kienast Y, et al. Ang-2-VEGF-A CrossMab, a novel bispecific human IgG1 antibody blocking VEGF-A and Ang-2 functions simultaneously, mediates potent antitumor, antiangiogenic, and antimetastatic efficacy. Clin Cancer Res. 2013;19(24):6730-6740.

15. Koh YJ, et al. Double antiangiogenic protein, DAAP, targeting VEGF-A and angiopoietins in tumor angiogenesis, metastasis, and vascular leakage. Cancer Cell. 2010;18(2):171-184.

16. Roche bid for Avastin follow-on suffers blow with trial failure [news release]. Zurich, Switzerland: Reuters; October 20, 2016. http://www.reuters. com/article/us-roche-results-trial/roche-bid-foravastin-follow-on-suffers-blow-with-trial-failureidUSKCN12K1X2. Accessed December 15, 2017.

17. Nasarre $P$, et al. Host-derived angiopoietin-2 affects early stages of tumor development and vessel maturation but is dispensable for later stages of tumor growth. Cancer Res. 2009;69(4):1324-1333.

18. Eklund L, Kangas J, Saharinen P. AngiopoietinTie signalling in the cardiovascular and lymphatic systems. Clin Sci. 2017;131(1):87-103.

19. Puri MC, Rossant J, Alitalo K, Bernstein A, Partanen $J$. The receptor tyrosine kinase TIE is required for integrity and survival of vascular endothelial cells. EMBO J.1995;14(23):5884-5891.

20. Sato TN, et al. Distinct roles of the receptor tyrosine kinases Tie-1 and Tie-2 in blood vessel formation. Nature. 1995;376(6535):70-74.

21. Savant S, et al. The orphan receptor Tie1 controls angiogenesis and vascular remodeling by differentially regulating Tie 2 in tip and stalk cells. Cell Rep. 2015;12(11):1761-1773.

22. D'Amico G, et al. Tie1 deletion inhibits tumor growth and improves angiopoietin antagonist therapy. J Clin Invest. 2014;124(2):824-834.

23. Korhonen EA, et al. Tie1 controls angiopoietin function in vascular remodeling and inflammation. J Clin Invest. 2016;126(9):3495-3510.

24. Kim M, et al. Opposing actions of angiopoietin-2 
on Tie2 signaling and FOXO1 activation. J Clin Invest. 2016;126(9):3511-3525.

25. Woo KV, et al. Tie1 attenuation reduces murine atherosclerosis in a dose-dependent and shear stress-specific manner. J Clin Invest. 2011;121(4):1624-1635.

26. Kaipainen A, et al. Enhanced expression of the tie receptor tyrosine kinase mesenger RNA in the vascular endothelium of metastatic melanomas. Cancer Res. 1994;54(24):6571-6577.

27. Hatva E, et al. Expression of endothelial cellspecific receptor tyrosine kinases and growth factors in human brain tumors. Am J Pathol. 1995;146(2):368-378.

28. Hatva E, Jääskeläinen J, Hirvonen H, Alitalo K, Haltia M. Tie endothelial cell-specific receptor tyrosine kinase is upregulated in the vasculature of arteriovenous malformations. J Neuropathol Exp Neurol. 1996;55(11):1124-1133.

29. Lin WC, et al. tie-1 protein tyrosine kinase: a novel independent prognostic marker for gastric cancer. Clin Cancer Res. 1999;5(7):1745-1751.

30. Aguayo A, et al. Clinical relevance of Flt1 and Tie1 angiogenesis receptors expression in B-cell chronic lymphocytic leukemia (CLL). Leuk Res. 2001;25(4):279-285.

31. Overwijk WW, Restifo NP. B16 as a mouse model for human melanoma. Curr Protoc Immunol. 2001; Chapter 20:Unit 20.1.

32. $\mathrm{Hu}$ J, et al. Endothelial cell-derived angiopoietin-2 controls liver regeneration as a spatiotemporal rheostat. Science. 2014;343(6169):416-419.

33. Felcht $\mathrm{M}$, et al. Angiopoietin-2 differentially regulates angiogenesis through TIE2 and integrin signaling. J Clin Invest. 2012;122(6):1991-2005.

34. del Toro R, et al. Identification and functional analysis of endothelial tip cell-enriched genes. Blood. 2010;116(19):4025-4033.

35. Hatva E, Böhling T, Jääskeläinen J, Persico MG, Haltia M, Alitalo K. Vascular growth factors and receptors in capillary hemangioblastomas and hemangiopericytomas. Am J Pathol. 1996;148(3):763-775.

36. Fife CM, et al. Stathmin mediates neuroblastoma metastasis in a tubulin-independent manner via RhoA/ROCK signaling and enhanced transendothelial migration. Oncogene. 2017;36(4):501-511.

37. Seegar TC, et al. Tie1-Tie2 interactions mediate functional differences between angiopoietin ligands. Mol Cell. 2010;37(5):643-655.

38. Saharinen $P$, et al. Multiple angiopoietin recombinant proteins activate the Tie1 receptor tyrosine kinase and promote its interaction with Tie2. JCell Biol. 2005;169(2):239-243.

39. Yuan HT, et al. Activation of the orphan endothelial receptor Tie1 modifies Tie2-mediated intracellular signaling and cell survival. FASEB J. 2007;21(12):3171-3183.

40. Thurston G, et al. Leakage-resistant blood vessels in mice transgenically overexpressing angiopoietin-1. Science. 1999;286(5449):2511-2514.

41. Thurston G, et al. Angiopoietin-1 protects the adult vasculature against plasma leakage. $\mathrm{Nat}$ Med. 2000;6(4):460-463.

42. Goel S, et al. Effects of vascular-endothelial protein tyrosine phosphatase inhibition on breast cancer vasculature and metastatic progression. J Natl Cancer Inst. 2013;105(16):1188-1201.

43. Lin P, et al. Antiangiogenic gene therapy targeting the endothelium-specific receptor tyrosine kinase Tie2. Proc Natl Acad Sci U S A. 1998;95(15):8829-8834.
44. Weis SM, Cheresh DA. Tumor angiogenesis: molecular pathways and therapeutic targets. Nat Med. 2011;17(11):1359-1370.

45. Loges S, Schmidt T, Carmeliet P. Mechanisms of resistance to anti-angiogenic therapy and development of third-generation anti-angiogenic drug candidates. Genes Cancer. 2010;1(1):12-25.

46. Holash J, et al. Vessel cooption, regression, and growth in tumors mediated by angiopoietins and VEGF. Science. 1999;284(5422):1994-1998.

47. Zagzag D, et al. In situ expression of angiopoietins in astrocytomas identifies angiopoietin-2 as an early marker of tumor angiogenesis. Exp Neurol.1999;159(2):391-400.

48. Scharpfenecker M, Fiedler U, Reiss Y, Augustin HG. The Tie-2 ligand angiopoietin-2 destabilizes quiescent endothelium through an internal autocrine loop mechanism. JCell Sci. 2005;118(Pt 4):771-780.

49. Peterson TE, et al. Dual inhibition of Ang-2 and VEGF receptors normalizes tumor vasculature and prolongs survival in glioblastoma by altering macrophages. Proc Natl Acad Sci U S A. 2016;113(16):4470-4475.

50. Kloepper J, et al. Ang-2/VEGF bispecific antibody reprograms macrophages and resident microglia to anti-tumor phenotype and prolongs glioblastoma survival. Proc Natl Acad Sci U S A. 2016;113(16):4476-4481.

51. Qu X, Tompkins K, Batts LE, Puri M, Baldwin HS, Baldwin S. Abnormal embryonic lymphatic vessel development in Tie1 hypomorphic mice. Development. 2010;137(8):1285-1295.

52. Wang Y, et al. Ephrin-B2 controls VEGF-induced angiogenesis and lymphangiogenesis. Nature. 2010;465(7297):483-486. 